\title{
Quality of life in cervical dystonia after treatment with botulinum toxin A: a 24-week prospective
} study

\author{
Subsai Kongsaengdao $0^{1,2}$ \\ Benchalak Maneeton ${ }^{3}$ \\ Narong Maneeton ${ }^{3}$ \\ 'Division of Neurology, Department \\ of Medicine, Rajvithi Hospital, \\ Department of Medical Services, \\ Public Health Ministry, Bangkok, \\ Thailand; ${ }^{2}$ Department of Medicine, \\ College of Medicine, Rangsit \\ University, Bangkok, Thailand; \\ ${ }^{3}$ Department of Psychiatry, Faculty \\ of Medicine, Chiang Mai University, \\ Thailand
}

This article was published in the following Dove Press journal:

Neuropsychiatric Disease and Treatment

10 January 2017

Number of times this article has been viewed

Objective: This study aimed to identify possible improvements in disease-specific health-related quality of life (HRQoL) after multiple injections of botulinum toxin A over 24 weeks in Thai cervical dystonia $(\mathrm{CD})$ patients.

Materials and methods: A 24-week prospective study comparing HRQoL of Thai CD patients before and after multiple injections of botulinum toxin A at 3-month intervals was performed. Disease-specific HRQoL was assessed by using the Cervical Dystonia Impact Profile-58 questionnaire (CDIP-58) and the Craniocervical Dystonia Questionnaire-24 (CDQ-24). General HRQoL was assessed by using the Medical Outcomes' 36-Item Short Form Health Survey (SF-36) and the EuroQoL 5-dimension questionnaire (EQ-5D). All the assessments were performed before and after the 24-week treatment period.

Results: A total of $20 \mathrm{CD}$ patients were enrolled in this study from April to December 2011. CDIP-58 and CDQ-24 scores, which assess disease-specific HRQoL, showed a significant improvement after 24 weeks of treatment by botulinum toxin A $(P<0.001)$. However, EQ-5D and SF-36 scores, which assess general HRQoL, showed no significant improvement after the treatment $(P>0.05)$.

Conclusion: CD patients' disease-specific HRQoL improved after being treated with multiple botulinum toxin A injections. However, general HRQoL was not improved.

Keywords: cervical dystonia, botulinum toxin A, the health-related quality of life, 36-Item Short Form Health Survey, EuroQoL 5-dimension questionnaire, Cervical Dystonia Impact Profile-58 questionnaire, Craniocervical Dystonia Questionnaire-24

\section{Introduction}

Cervical dystonia (CD) is a common abnormal movement disorder, in which patients suffer from repetitive and/or sustained involuntary contractions of neck muscles, resulting in abnormal neck twisting and/or posture. The causes of CD may or may not be identifiable. ${ }^{1} \mathrm{CD}$ can be an immensely painful, distressing, and hopeless experience for sufferers with contingent mental disorder. The common types of CD are lateral flexion or tilt of the neck (laterocollis), posterior extension of the neck (retrocollis), forward flexion of the neck (anterocollis), and horizontal turning of the neck (torticollis). ${ }^{1,2}$ $\mathrm{CD}$ is found in one in every 250,000 people in Rochester, Minnesota, USA, and 5.7 in 100,000 in a European study. ${ }^{1}$ The worldwide estimate of CD incidents is 0.80 per 100,000 person-years and is most commonly found among Caucasian women. ${ }^{3}$ Most CD cases develop when patients are in their 50 s.

The etiology of CD may be unidentifiable (primary CD) or secondary to other neurological diseases (secondary CD) such as post encephalitis syndrome, post stroke
Correspondence: Benchalak Maneeton Department of Psychiatry, Faculty of Medicine, Chiang Mai University, I I 0 Intavaroros Road, Si Phum, Muang, Chiang Mai 50200, Thailand Tel +66 53935422 Fax +6653935426 Email benchalak.maneeton@cmu.ac.th 
dystonia, and other neurodegenerative diseases that cause an organic damage to the basal ganglia. ${ }^{1,2}$ Functional magnetic resonance imaging of CD patients shows an increased activation of putamen, thalamus, and caudate nucleus. ${ }^{3,4} \mathrm{~A}$ study in a primate model of CD demonstrates that destruction of GABA-nergic neuron in the brainstem, basal ganglia, and thalamus may cause CD with sensory trick, explaining the aberrant sensory representations interfering motor control. ${ }^{2}$ Stress and fatigue may exacerbate CD, whereas relaxation, sleep, and sensory movements may improve the symptoms. ${ }^{1}$

Most of the oral medications including anticholinergics, benzodiazepines, and antispasmodics and surgical treatments including selective peripheral denervation and deep brain stimulation have been shown ineffective in the treatment of CD. Most CD patients who receive medications such as trihexyphenidyl, carbamazepine, clonazepam, and baclofen report sustained symptoms and suffer from unpleasant side effects. ${ }^{2,3,5,6}$ Interestingly, intramuscular injections of botulinum toxin A offer higher efficacy and fewer side effects than other treatments. ${ }^{5-14}$ Botulinum toxin $\mathrm{A}$ is a highly potent neurotoxin that is used to treat various diseases including hemifacial spasm, spasticity, dystonia, and achalasia. Treatment by botulinum toxin $\mathrm{A}$ is shown to improve the quality of life of CD patients. ${ }^{3,5-16}$ Botulinum toxin A is approved by the Food and Drug Administration of the United States for $\mathrm{CD}$ treatment and is recommended by most guidelines as a first-line treatment worldwide.

CD needs accurate assessment of treatment outcomes. ${ }^{17,18}$ In $\mathrm{CD}$ patients, psychiatric features and pain are important determinants of disability. Interventions to reduce psychiatric problems and pain should have a more prominent role in the treatment of CD patients in order to improve disability levels. ${ }^{19}$ Health-related quality of life (HRQoL) is a useful evaluation of general well-being of patients with regard to physical health, mental state, and social function. Generally, an improvement in physical conditions enhances the HRQoL. Improved HRQoL in CD patients may be due to improved vision interference during reading and writing, social embarrassment, pain, depression, and anxiety.

The effectiveness of CD treatment by botulinum toxin A injections has not been studied in Thai patients. Many questionnaires are used to assess general and disease-specific HRQoL of CD patients before and after treatment with botulinum toxin A injections. Medical Outcomes' 36-Item Short Form Health Survey (SF-36) and the EuroQoL 5-dimension questionnaire (EQ-5D) are used to assess general HRQoL while the Cervical Dystonia Impact Profile-58 questionnaire (CDIP-58) and the 24-question Craniocervical Dystonia Questionnaire (CDQ-24) are used to assess diseasespecific HRQoL. ${ }^{13,16,18,20-24}$

\section{Materials and methods}

This research had the approval of the Rajavithi Hospital, Department of Medical Services, Public Health Ministry, Bangkok, Thailand ethics committee.

Male and female patients aged between 18 and 80 years who fulfilled the diagnostic criteria for primary CD from April to December 2011, had good consciousness and comprehension of the HRQoL questionnaires, and could communicate in and understand Thai language were included in the study. Patients who were $<18$ years of age, could not understand Thai language, declined consent to join the study, and were potentially pregnant or lactating were excluded. Patients with other medical conditions that could influence trial assessments, for example, bleeding abnormalities, arthritis, heart disease, and other neurological and psychological disease, were also excluded. Twenty patients with CD were screened and invited to the study. All the patients were included and completed the study.

\section{The disease-specific HRQoL questionnaires}

To assess the disease-specific HRQoL, this study used the CDIP-58 and CDQ-24.

\section{CDIP-58}

CDIP-58 is a validated patient-based scale for health outcomes of patients with CD. It comprises eight health dimensions with proven good reliability testing and has been used to measure health impacts of CD. ${ }^{18}$ The eight subscales include head and neck symptoms, pain and discomfort, upper limb activities, walking, sleep, annoyance, mood, and psychosocial functioning. ${ }^{20,24}$ The Thai language translation of CDIP-58 has previously been tested at Rajavithi Hospital for its consistency in comparison with the original CDIP-58, resulting in Cronbach's alpha of $>0.7$.

\section{CDQ-24}

CDQ-24 is a validated disease-specific questionnaire designed to evaluate HRQoL of CD and blepharospasm patients. It has been shown to be appropriate for use in both clinical trials and clinical practice with stable internal consistency for all preliminary subscales ( $\alpha>0.7$ each). The 24 items of CDQ-24 comprise five categories, which are stigma (questions $7,8,9,10,18,22$ ), emotional well-being 
(questions 11, 12, 13, 14, 15), pain (questions 4, 5, 21), activities of daily living (ADL) (questions 1, 2, 3, 6, 19, 20), and social and family life (questions $16,17,23,24) .{ }^{16,24}$ Thai translation of the original CDQ-24 has previously been tested at Rajavithi Hospital to ensure its conformity to the original CDIP-58, resulting in Cronbach's alpha of $>0.7$.

\section{The general HRQoL questionnaires}

This study employed the SF-36 and the EQ-5D to assess patients' general HRQoL.

\section{SF-36}

SF-36 is a 36-item questionnaire that consists of eight domains: physical functioning (PF), role limitations due to physical health (RP), role limitations due to emotional problems (RE), vitality (VT), mental health, social functioning (SF), bodily pain (BP), and general health $(\mathrm{GH})$. PF, RP, and $\mathrm{BP}$ domains comprise the physical health half of the questionnaire while RE, VT, mental health, and SF the mental health. The Thai language version of SF-36 has been validated and tested for its reliability in Thai patients..$^{21,22,24}$

\section{EQ-5D}

EQ-5D is a short five-item questionnaire to assess the GH outcome after treatments. EQ-5D is a simple survey questionnaire often used in general clinics. It is cognitively simple. It has a short completing time and instructions to respondents included in the questionnaire, which makes it useful for screening. The EQ-5D comprises five dimensions, which are mobility, self-care, usual activities, pain/discomfort, and anxiety/depression. Each dimension has three levels of potential outcomes: no problems, some problems, and extreme problems. ${ }^{23,24}$ The EQ-5D (Thai version) has also been validated and tested for reliability in Thai patients. ${ }^{25}$

\section{Study design}

This study is a 24-week prospective assessment of the quality of life of CD patients who were treated with botulinum toxin A injections. Patients who met all inclusion criteria and no exclusion criteria were enrolled in the study. They filled the four questionnaires at week 0 before the first injection and at week 24 after the follow-up injection. All patients were trained to self-evaluate intensity scores on a 5-point scale.

Botulinum toxin A, which was supplied as a freeze-dried powder, was diluted in normal saline, and used within $2 \mathrm{~h}$ of preparation. All enrolled patients received intramuscular injections in the following neck muscles: sternocleidomastoid, trapezius, and splenius capitis muscles at the opposing side (Figure 1). Each patient was assessed agonist/antagonist spasm of the sternocleidomastoid, trapezius and splenius capitis muscles to determine the site of injection. Totally 100 units of botulinum toxin A were resuspended in $3 \mathrm{~mL}$ of normal saline, resulting in solution of $\sim 33.33$ units of botulinum toxin A per $\mathrm{mL}$. Each patient was injected 5-10 units $(0.15-0.30 \mathrm{~mL})$ into each affected site of muscles by using $29 \mathrm{G}$ insulin needles. However, if each muscle has several affected sites, then the total botulinum toxin was 50 units per injection. All patients gave written informed consent before the start of the treatment.

\section{Assessments}

Two assessments were performed during the course of the study. The first (baseline) assessment was performed at week 0 before the first injection, and the second at week 24 during the follow-up treatment (Figure 2). At each visit, patients underwent physical and neurological examinations and completed four HRQoL questionnaires, CDIP-58, CDQ-24, SF-36, and EQ-5D.

\section{Quality of life endpoints}

The primary endpoints were the mean change from baseline of CDIP-58 and CDQ-24 scores. The secondary endpoints were the change in the mean score of SF-36 and EQ-5D questionnaire results.

\section{Statistical analysis}

Descriptive statistical analysis was used for demographic data. The mean (standard deviation [SD]) scores of CDIP-58, CDQ-24, SF-36, and EQ-5D at week 0 and week 24 were compared by the Wilcoxon signed-rank test. Cytel $^{\circledR}$ Studio $^{\circledR}$ (license no 2060107) software package was used in data analysis. Results with $P<0.05$ are deemed significant. Sample size estimation was not performed because there is

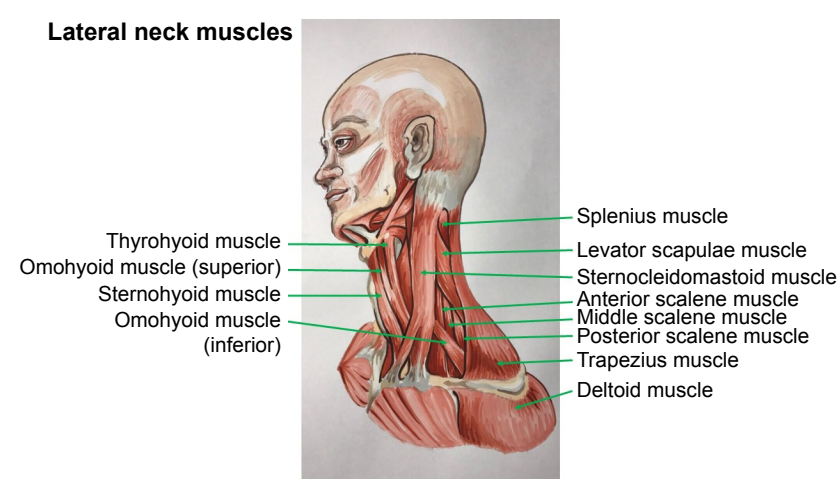

Figure I Lateral neck muscles. 


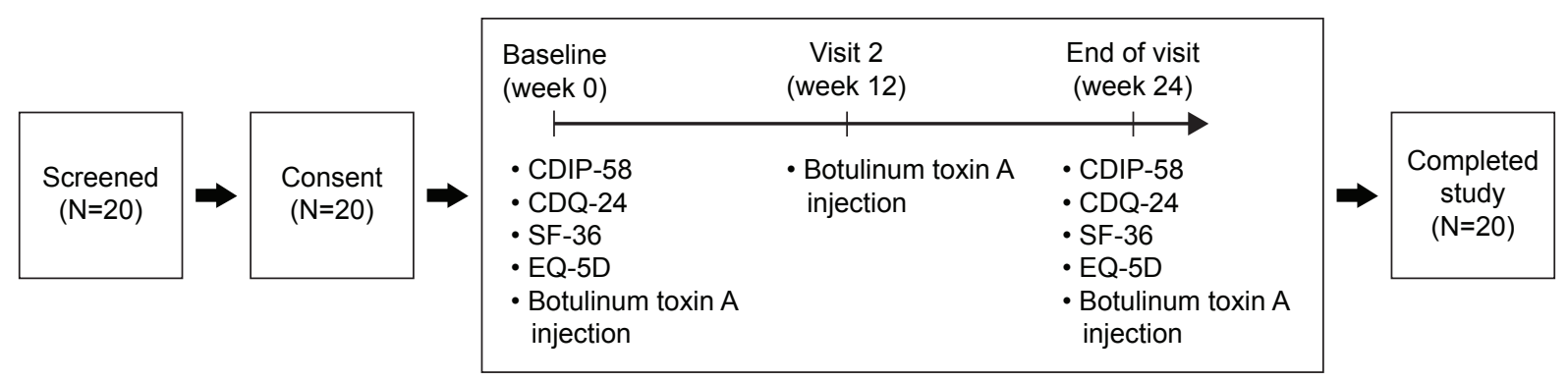

Figure 2 Schematic diagram showing progression of 20 subjects from screening.

Abbreviations: CDIP-58, Cervical Dystonia Impact Profile-58 questionnaire; CDQ-24, Craniocervical Dystonia Questionnaire-24; EQ-5D, EuroQoL 5-dimension questionnaire; SF-36, 36-Item Short Form Health Survey.

no previous study regarding HRQoL in CD patients treated with botulinum toxin $\mathrm{A}$.

\section{Results}

A total of 13 females and 7 males of age ranging from 24 to 75 years (mean $[\mathrm{SD}]=54.8[12.5]$ years) were enrolled in the study from April to December 2011. All enrolled patients have been included in the analysis. Mean duration of symptoms in enrolled patients was 5.0 years (range from 0 to 12 years). There were 16 cases of torticollis, two of laterocollis, one of anterocollis, and one of latrocollis included in the study. The previous mean number of botulinum toxin A injections received per patient was $8.85 \pm 5.32$ injections, ranging from 1 to 22. Demographic data are shown in Table 1.

\section{Primary outcomes: the disease-specific HRQoL}

After 24 weeks of treatment, the mean (SD) score of all eight domains of CDIP-58 including head and neck symptoms, pain and discomfort, upper limb activities, walking, sleep, annoyance, mood, and psychosocial functioning were significantly improved $(P<0.001)$ (Table 2$)$. The mean (SD) of each of the five categories of CDQ-24 was also

Table I Demographic and baseline clinical characteristics of patients with cervical dystonia

\begin{tabular}{ll}
\hline Demographics & \\
\hline Number & 20 \\
Gender & $7(35 \%)$ \\
$\quad$ Male & $13(65 \%)$ \\
Female & $54.8(12.5)$ \\
Mean (SD) age (years) & $5.0(3.1)$ \\
Mean (SD) disease duration (years) & \\
Type of cervical dystonia $n(\%)$ & $16(80)$ \\
$\quad$ Torticollis & $2(10)$ \\
Laterocollis & $1(5)$ \\
Anterocollis & $1(5)$ \\
$\quad$ Retrocollis & $50.0(0.0)$ \\
Mean (SD) BTX injection dosage & $8.9(5.3)$ \\
Previous mean (SD) BTX injection time
\end{tabular}

Abbreviations: BTX, botulinum toxin; SD, standard deviation. significantly improved with $P$-values of $0.004,0.001,0.003$, 0.001, and 0.0005 for stigma, emotional well-being, pain, activities of daily living, and social and family life, respectively (Table 2).

\section{Secondary outcomes: general HRQoL}

After 24 weeks of treatment, the mean values of results of all eight domains of SF-36 did not significantly improved. The $P$-values for PF, RP, RE, VT, mental health, SF, BP, and $\mathrm{GH}$ are $0.726,1.000,0.505,0.349,0.115,0.171,0.427$, and 0.380 respectively. The mean score of each category of EQ-5D including mobility, self-care, usual activities, pain/ discomfort, and anxiety/depression revealed a nonsignificant change $(P>0.05)$ (Table 2).

\section{Discussion}

The comparison of CD patients' quality of life pre- and posttreatments by either medical or surgical measures has been performed but not in Thai patients. ${ }^{1-3,26-29}$ This prospective study employed four questionnaires to compare the HRQoL of CD patients before and after treatment with botulinum toxin A injections. With respect to the primary endpoint, disease-specific HRQoL (CDIP-58 and CDQ-24) scores were significantly improved in all sub-categories $(P<0.001)$, and both the questionnaires showed a good correlation. Similar results have been shown in previous studies with botulinum toxin A treatment. ${ }^{1-16,18,20}$ The secondary endpoints (SF-36 and EQ-5D) were not statistically significant in improvement in all subscales.

CDIP-58 and CDQ-24 are two disease-specific HRQoL questionnaires that have been developed and established to use to assess the quality of life of $\mathrm{CD}$ patients worldwide. Both the questionnaires, originally in English, have been well validated. ${ }^{16,18,20,24}$ They were translated into Thai language and tested at Rajavithi Hospital. Both have been shown to be consistent with the original questionnaires with Cronbach's alpha $>0.7$. 
Table 2 Clinical score before (week 0) and after (week 24) treatment as measured by CDIP-58, CDQ-24, SF-36, and EQ-5D, mean (SD)

\begin{tabular}{|c|c|c|c|}
\hline Questionnaires & $\begin{array}{l}\text { Score at week } 0 \\
\text { (baseline) mean (SD) }\end{array}$ & $\begin{array}{l}\text { Score at week } 24 \\
\text { mean (SD) }\end{array}$ & $P$-value \\
\hline \multicolumn{4}{|l|}{ CDIP-58 } \\
\hline Head and neck symptoms (6 items) & I6.I (2.3) & $9.5(2.7)$ & $<0.001$ \\
\hline Pain and discomfort (5 items) & $18.2(2.7)$ & $10.4(2.6)$ & $<0.001$ \\
\hline Upper limb activity (9 items) & $26.8(6.2)$ & $15.3(4.7)$ & $<0.001$ \\
\hline Walking (9 items) & $26.6(6.5)$ & $15.4(5.9)$ & $<0.001$ \\
\hline Sleep (4 items) & $13.4(3.4)$ & $7.4(2.6)$ & $<0.001$ \\
\hline Annoyance (8 items) & $22.7(4.3)$ & $13.5(3.8)$ & $<0.001$ \\
\hline Mood (7 items) & I5.7 (5.0) & $9.4(2.4)$ & $<0.001$ \\
\hline Psychosocial functioning ( 10 items) & $25.0(6.8)$ & I6.I (5.5) & $<0.001$ \\
\hline \multicolumn{4}{|l|}{ CDQ-24 } \\
\hline Stigma & $15.3(6.6)$ & $9.3(3.1)$ & 0.004 \\
\hline Emotional well-being & II.8 (4.7) & $6.8(2.1)$ & 0.001 \\
\hline Pain & $7.9(2.8)$ & $4.6(2.4)$ & 0.003 \\
\hline Activities of daily living & $15.9(5.8)$ & $9.9(3.1)$ & 0.001 \\
\hline Social and family life & $7.0(1.7)$ & $4.6(0.9)$ & $<0.001$ \\
\hline \multicolumn{4}{|l|}{ SF-36 } \\
\hline Physical functioning & $54.7(38.2)$ & $61.6(25.3)$ & 0.726 \\
\hline Role limitations due to physical health & $57.8(45.4)$ & $57.8(36.2)$ & 1.000 \\
\hline Role limitations due to emotional problems & $66.7(43.9)$ & $75.0(35.5)$ & 0.505 \\
\hline Vitality & $58.1(22.6)$ & $61.3(14.3)$ & 0.349 \\
\hline Mental health & $63.0(22.1)$ & $71.5(14.1)$ & 0.115 \\
\hline Social functioning & $76.5(21.0)$ & $83.8(13.7)$ & 0.171 \\
\hline Bodily pain & $58.8(30.5)$ & $62.5(25.4)$ & 0.427 \\
\hline General health & $47.7(17.5)$ & $50.6(12.8)$ & 0.380 \\
\hline \multicolumn{4}{|l|}{ EQ-5D } \\
\hline Mobility & $\mathrm{I} .500(0.610)$ & $\mathrm{I} .375(0.500)$ & 0.157 \\
\hline Self-care & $1.313(0.602)$ & I.563 (0.727) & 0.102 \\
\hline Usual activities & $1.750(0.683)$ & $1.750(0.683)$ & 1.000 \\
\hline Pain/discomfort & $1.875(0.619)$ & $1.875(0.619)$ & 1.000 \\
\hline Anxiety/depression & $1.750(0.447)$ & $1.625(0.500)$ & 0.157 \\
\hline
\end{tabular}

Abbreviations: CDIP-58, Cervical Dystonia Impact Profile-58 questionnaire; CDQ-24, Craniocervical Dystonia Questionnaire-24; EQ-5D, EuroQoL 5-dimension questionnaire; SF-36, 36-Item Short Form Health Survey; SD, standard deviation.

The results of SF-36 and EQ-5D questionnaires were not significantly improved after treatment with botulinum toxin A. This may be due to the low sensitivity of SF-36 and EQ-5D. The SF-36 and EQ-5D may be suitable for the evaluation of general HRQoL; however, they may not be sensitive enough in detecting an improvement in HRQoL of CD patients compared to disease-specific questionnaires CDIP-58 and CDQ-24.

\section{Limitations}

The main limitation of this study was the small sample size. It has been suggested to recruit a larger subject group in future studies. Furthermore, a double-blind randomized placebo-controlled study is proposed to compare the primary outcomes such as the disease-specific QoL among the different commercial brands of Botulinum toxin (Ona-botulinum toxin A, Abo-botulinum toxin A, and Neu-botulinum toxin A). Further investigation with a larger sample size is warranted in order to account for individual differences, which may mask or exaggerate the outcomes of the study.

Botulinum toxin A is a zinc endopeptidase that acts on snare proteins in the pre-synaptic nerve terminal. Botulinum toxin A inhibits acetylcholine release by blocking calcium influx into pre-synaptic junction, resulting in focal denervation of neuromuscular junctions and loss of electrical activity in muscle action potential units. ${ }^{29}$ As shown in the results of this study, a significant improvement of HRQoL in CD patients after two consecutive injections with botulinum toxin $\mathrm{A}$ is predominantly due to an improvement in mental health rather than physical health. Botulinum toxin A itself does not affect the role of central neurotransmitter and is used only locally and in a small amount in this study. Therefore, an improvement in mental health is more likely a result of an improvement of physical health rather than a direct neural effect of the agent. 


\section{Conclusion}

In conclusion, the quality of life of $\mathrm{CD}$ patients improved after multiple botulinum toxin A injections. Each injection of botulinum toxin A seems to have cumulative effects in multiple-injection patients. The direct mechanism behind an improvement in physical health is well understood; however, that behind mental health is less obvious. A relief of dystonia could make a positive difference to patients' relaxation state and stress level, which would account for mental improvement. Disease-specific HRQoL questionnaires are better than general HRQoL questionnaires when the sample size is small.

\section{Acknowledgments}

We thank Professor Stephen D Martin, MBBS, MRCPsych, Honorary Professor of Psychiatry, University of Chiang Mai, for his substantial help in this project. We thank Medytox, Korea, and Celeste, Thailand, for supplying Neubotulinum toxin A $\left(\right.$ Neuronox $\left.^{\circledR}\right)$ used in this study.

\section{Disclosure}

The authors report no conflicts of interest in this work.

\section{References}

1. Coelh M, Valadas AF, Mestre T, Ferreira JJ. Pain and quality of life in the treatment of cervical dystonia. Eur Neurol Rev. 2009;4(2):74-78.

2. Geyer HL, Bressman SB. The diagnosis of dystonia. Lancet Neurol. 2006; 5(9):780-790.

3. Brashear A. The botulinum toxins in the treatment of cervical dystonia. Semin Neurol. 2001;21(1):85-90.

4. Marras C, Van den Eeden SK, Fross RD, et al. Minimum incidence of primary cervical dystonia in a multiethnic health care population. Neurology. 2007;69(7):676-680.

5. Costa J, Espirito-Santo C, Borges A, Ferreira JJ, Coelho M, Sampaio C. Botulinum toxin type A versus anticholinergics for cervical dystonia. Cochrane Database Syst Rev. 2005;(1):CD004312.

6. Brans JW, Lindeboom R, Snoek JW, et al. Botulinum toxin versus trihexyphenidyl in cervical dystonia: a prospective, randomized, doubleblind controlled trial. Neurology. 1996;46(4):1066-1072.

7. Walker FO. Botulinum toxin therapy for cervical dystonia. Phys Med Rehabil Clin N Am. 2003;14(4):749-766, vi.

8. Jankovic J. Treatment of dystonia. Lancet Neurol. 2006;5(10):864-872.

9. Simpson DM, Blitzer A, Brashear A, et al. Assessment: botulinum neurotoxin for the treatment of movement disorders (an evidencebased review): report of the Therapeutics and Technology Assessment Subcommittee of the American Academy of Neurology. Neurology. 2008;70(19):1699-1706.

10. Ferreira JJ, Costa J, Coelho M, Sampaio C. The management of cervical dystonia. Expert Opin Pharmacother. 2007;8(2):129-140.
11. Slawek J, Friedman A, Potulska A, et al. Factors affecting the healthrelated quality of life of patients with cervical dystonia and the impact of botulinum toxin type A injections. Funct Neurol. 2007;22(2):95-100.

12. Pekmezovic T, Svetel M, Ivanovic N, et al. Quality of life in patients with focal dystonia. Clin Neurol Neurosurg. 2009;111(2):161-164.

13. Hakkinen A, Ylinen J, Rinta-Keturi M, Talvitie U, Kautiainen H, Rissanen A. Decreased neck muscle strength is highly associated with pain in cervical dystonia patients treated with botulinum toxin injections. Arch Phys Med Rehabil. 2004;85(10):1684-1688.

14. Hilker R, Schischniaschvili M, Ghaemi M, Jacobs A, Rudolf J. Health related quality of life is improved by botulinum neurotoxin type A in long term treated patients with focal dystonia. J Neurol Neurosurg Psychiatry. 2001;71(2):193-199.

15. Muller J, Wissel J, Kemmler G, et al. Craniocervical dystonia questionnaire (CDQ-24): development and validation of a disease-specific quality of life instrument. J Neurol Neurosurg Psychiatry. 2004;75(5): 749-753.

16. Colosimo C, Pantano P, Calistri V, Totaro P, Fabbrini G, Berardelli A. Diffusion tensor imaging in primary cervical dystonia. $J$ Neurol Neurosurg Psychiatry. 2005;76(11):1591-1593.

17. Cano SJ, Warner TT, Linacre JM, et al. Capturing the true burden of dystonia on patients: the Cervical Dystonia Impact Profile (CDIP-58). Neurology. 2004;63(9):1629-1633.

18. Cano SJ, Hobart JC, Edwards M, et al. CDIP-58 can measure the impact of botulinum toxin treatment in cervical dystonia. Neurology. 2006; 67(12):2230-2232.

19. van den Dool J, Tijssen MA, Koelman JH, Engelbert RH, Visser B. Determinants of disability in cervical dystonia. Parkinsonism Relat Disord. Epub 2016 Aug 15.

20. Leurmarnkul W. Properties testing of the retranslated SF-36 (Thai version). Thai J Pharm Sci. 2005;29(1-2):69-88.

21. Ware JE Jr. SF-36 health survey update. Spine. 2000;25(24):3130-3139.

22. Sakthong P, Kasemsup V. Health-related quality of life in Thai peritoneal dialysis patients. Asian Biomed. 2011;5(6):799-805.

23. Herndon RM. Handbook of Neurologic Rating Scales. New York: Demos; 2006.

24. Cohen-Gadol AA, Ahlskog JE, Matsumoto JY, Swenson MA, McClelland RL, Davis DH. Selective peripheral denervation for the treatment of intractable spasmodic torticollis: experience with 168 patients at the Mayo Clinic. J Neurosurg. 2003;98(6):1247-1254.

25. Tongsiri S. The Thai Population-Based Preference Scores for EQ-5D Health States. London: London School of Hygiene and Tropical Medicine, University of London; 2009.

26. Munchau A, Palmer JD, Dressler D, et al. Prospective study of selective peripheral denervation for botulinum-toxin resistant patients with cervical dystonia. Brain. 2001;124(Pt 4):769-783.

27. Kiss ZH, Doig-Beyaert K, Eliasziw M, et al. The Canadian multicentre study of deep brain stimulation for cervical dystonia. Brain. 2007; 130(Pt 11):2879-2886.

28. Delnooz CC, Horstink MW, Tijssen MA, van de Warrenburg BP. Paramedical treatment in primary dystonia: a systematic review. Mov Disord. 2009;24(15):2187-2198.

29. Tan NC, Chan LL, Tan EK. Hemifacial spasm and involuntary facial movements. QJM. 2002;95(8):493-500.
Neuropsychiatric Disease and Treatment

\section{Publish your work in this journal}

Neuropsychiatric Disease and Treatment is an international, peerreviewed journal of clinical therapeutics and pharmacology focusing on concise rapid reporting of clinical or pre-clinical studies on a range of neuropsychiatric and neurological disorders. This journal is indexed on PubMed Central, the 'PsycINFO' database and CAS,

\section{Dovepress}

and is the official journal of The International Neuropsychiatric Association (INA). The manuscript management system is completely online and includes a very quick and fair peer-review system, which is all easy to use. Visit http://www.dovepress.com/testimonials.php to read real quotes from published authors. 